\title{
Espessamento em fi de parede celular
}

\author{
Hyrandir Cabral de Melo ${ }^{1}$
}

Recebido: 15.09.2010; aceito: 3.03.2011

\begin{abstract}
Phi thickening of cell wall). Phi thickening occurs since epidermis till the endodermis' adjacent layer in roots of various angiosperm and gymnosperm species. Unlikely Casparian strips they are not suberized and they can be found in varied points of the cell wall in a unique cell. In some species the phi thickening development can be observed since the first centimeter from root apex. Phi thickening has received the role of mechanical support to tissues in radial expansion process and roots in longitudinal growing in substrates with high level of difficulty to root penetration, also as physical barrier against the fungi invasion to the cortex deep layers or to the roots central cylinder and also to ions selection which moves by apoplastic way. Instead phi thickenings continue being used as a root anatomic character to taxonomic studies of some species, it has been proven that its development is not necessarily constitutive, what can makes it improper for this use. Key words: Casparian strips, ions uptake, mycorrhiza, phi layer
\end{abstract}

RESUMO - (Espessamento em fi de parede celular). Os espessamentos em fi ocorrem desde a epiderme até a camada adjacente à endoderme em raízes de várias espécies de angiospermas e gimnospermas. Diferentemente das estrias de Caspary, não são suberizados e podem ser localizados em variados pontos da parede celular de uma mesma célula. Em algumas espécies o seu desenvolvimento pode ser observado já no primeiro centímetro do ápice radicular. Funcionalmente, têm sido atribuídos ao espessamento em fi papéis de sustentação mecânica para suporte dos tecidos em processo de expansão radial e de crescimento longitudinal de raízes em substratos com alto grau de impedimento à sua penetração, de barreira física contra a invasão de fungos em camadas mais profundas do córtex ou no cilindro central e de seleção de íons que se movimentam por via apoplástica. Embora o espessamento em fi continue sendo usado como um caractere anatômicoradicular para estudos de taxonomia de algumas espécies, tem sido comprovado que seu desenvolvimento não é necessariamente constitutivo, o que pode torná-lo inadequado para este fim.

Palavras-chave: absorção de íons, estrias de Caspary, metais pesados, micorriza

\section{Introdução}

Alguns espessamentos lignificados de parede celular têm sido reportados na literatura botânica atual, no entanto muitos continuam sendo desconhecidos quanto a sua função, caso especial do espessamento em fi, que embora seja conhecido e estudado há mais de um século, até hoje é relatado como uma estrutura enigmática do ponto de vista funcional, embora haja evidências de sua multifuncionalidade, tanto para aspectos estruturais quanto fisiológicos. Na busca de se estabelecer uma função para o espessamento em fi foi desenvolvido um vasto conhecimento envolvendo esta estrutura que integra estudos de paleontologia, taxonomia, morfologia e ultra-estrutura, ecologia, interação planta-microrganismos e fisiologia vegetal.
Este artigo tem o objetivo de explorar a formação, desenvolvimento, caracterização, funcionalidade e usos dos espessamentos em fi de parede celular através de um apanhado dos estudos científicos realizados desde a época de sua descoberta até os dias atuais.

\section{Características e localização}

Espessamentos em fi geralmente ocorrem na parede radial e tangencial de células radiculares, preferencialmente corticais, de algumas espécies de gimnospermas e angiospermas, mas sua ocorrência também foi registrada nas paredes anticlinaistransversais e periclinais nos septos radiais da raiz e caule das macrófitas aquáticas Bacopa salzmanni

1. Universidade Federal de Goiás, Instituto de Ciências Biológicas I, Departamento de Biologia Geral (Botânica), Campus Samambaia, 74001-970 Goiânia, GO, Brasil. hyrandir@yahoo.com.br. 
(Benth.) Wettst. ex Edwall e Bacopa monnierioides (Cham.) Robinson (Bona \& Morretes 2003). Sua denominação advém da semelhança de sua forma à letra grega $\Phi$, vista em seção transversal e em duas células contíguas (Russow 1875).

Van Tieghem (1888), observando o espessamento em fi em diferentes gêneros e espécies de gimnospermas, classificou o espessamento em fi em três tipos, de acordo com sua localização no córtex radicular. O tipo I, e o mais comum, se localiza na camada celular adjacente à endoderme; o tipo II se localiza na camada celular cortical adjacente à epiderme, e o tipo III, se localiza nas demais camadas celulares do córtex. Haas et al. (1976) demonstraram que o espessamento em fi ocorre na hipoderme radicular de Pelargonium hortorum Bailey, e Degenhardt \& Gimmler (2000) demonstraram sua ocorrência na rizoderme de Zea mays $\mathrm{L}$.

\section{Composição química e ultraestrutura}

A comparação entre espessamento em fi e estrias de Caspary tem sido quase que uma constante desde o início de suas investigações, no intuito de compará-los quanto as suas funcionalidades. Enquanto as estrias de Caspary contêm suberina e lignina associadas à sua estrutura celulósica, ou somente suberina (Peterson \& Perumala 1990, Wilson \& Peterson 1983), o espessamento em fi contém apenas lignina (Degenhardt \& Gimler 2000, Haas et al. 1976, Mackenzie 1979, Pratikakis et al. 1998, Reidhart \& Guard 1957), exceção feita aos espessamentos em fi encontrados em Pyrus communis L., destituídos não somente de suberina, mas também de lignina (Esau 1943). Uma consequência direta dessa diferença de composição química entre as duas estruturas é quanto a permeabilidade de parede, uma vez que a suberina é uma substância reconhecidamente hidrofóbica.

Além do espessamento em fi diferir das estrias de Caspary quanto a permeabilidade de parede, Haas et al. (1976) também observaram em raízes de Pelargonium hortorum Bailey que o espessamento em fi pode ocorrer em várias regiões da parede de uma mesma célula, desenvolvendo-se nos pontos de contato desta com outras células. Estes pesquisadores também observaram que não há ocorrência de plasmodesmos na região fi-espessada, mas há associação de microtúbulos aos espessamentos e observaram também que não há aderência da plasmalema às porções fi-espessadas de parede. Todas essas características diferem daquelas encontradas nas estrias de Caspary, as quais foram analisadas concomitantemente. No entanto, essas características não podem ser generalizadas, uma vez que em Brassica oleracea L. cv. Maratón, Fernandez-Garcia et al. (2009) observaram a presença de muitos plasmodesmos atravessando a região de parede fiespessada e Bona (1999) registrou a presença de pontoações na região do espessamento em Bacopa salzmanni Wettst. ex Edwall e Bacopa monnierioides Cham.

As evidências ultra-estruturais, de permeabilidade e a localização do espessamento em fi em tecidos que ocorrem desde a epiderme até camadas adjacentes a endoderme, podendo essa ocorrência ser simultânea em múltiplas camadas celulares, além de variáveis espessamentos por célula (Peterson \& Choleva 1998, Pires et al. 2003), distinguem o espessamento em fi das estrias de Caspary, levando pesquisadores a refutarem a idéia de que ambos teriam funções estritamente similares (Gerrath et al. 2002, Pratikakis et al. 1998).

\section{Desenvolvimento}

Estudos relacionados ao desenvolvimento do espessamento em fi têm sido desenvolvidos na tentativa de esclarecer o enigmático papel funcional de sua estrutura. Pratikakis et al. (1998) relataram que já era possível a observação de espessamento em fi em seções de 0-1 cm do ápice radicular de Ceratonia siliqua L., identificados em uma ou duas camadas, adjacentes a endoderme, porém apenas em porções frontais a localização do floema. De 1-4 cm, região em que o xilema já estava bem diferenciado, um completo cilindro de até duas camadas de células com espessamento em fi já havia se formado no córtex. Os espessamentos em fi se desenvolveram e sofreram lignificação antes do início do processo de suberização da endoderme. Além destes pesquisadores, Wendenburg \& Peterson (1983), estudando Pyrus malus L., também relataram o completo desenvolvimento dos espessamentos em fi em seções de 3-5 mm do ápice radicular e não observaram a presença de espessamento em fi em raízes em estágio de crescimento secundário, e Pan et al. (2006), estudando o desenvolvimento de espessamento em fi em raízes de Eriobotrya japonica Lindl., observaram o início do seu desenvolvimento a $10 \mathrm{~mm}$ do ápice radicular, atingindo o máximo desenvolvimento a 
$30 \mathrm{~mm}$, e portanto, bem antes do completo desenvolvimento das estrias de Caspary.

Wenderburg \& Peterson (1983) observaram que há um prévio desaparecimento dos espessamentos em fi em zonas corticais de emergência das raízes laterais em Pyrus malus L., evidenciando digestão enzimática, assim como a formação desses espessamentos nas raízes laterais em formação antes mesmo de sua protrusão. Tajima et al. (2008) também observaram a presença de espessamento em fi em células corticais de raízes laterais de segunda e terceira ordens em Arachis hypogaea L. Para esta espécie as células que apresentavam espessamento em fi em suas paredes, quando em processo de crescimento secundário de raiz, mudaram suas formas de tabulares para oblongas irregulares, indicando que estas se encontravam sob pressão exercida pela expansão do cilindro central.

\section{Espessamento em fi e movimento apoplástico de íons}

Russow (1875) e Van Tieghem (1888) atribuíram ao espessamento em fi a função de sustentação mecânica ao córtex radicular, porém sem especificar a qual força esse suporte mecânico deveria se opor. Essa lacuna levou Boreau (1939) e Mackenzie (1979) a sugerirem que o espessamento em fi pudesse atuar de maneira similar às estrias de Caspary e, portanto, regular o fluxo de água e íons para o cilindro central da raiz, baseando-se, para isto, apenas na semelhança estrutural. Essa semelhança estrutural levou Van Fleet (1961) a considerar as camadas celulares fi-espessadas como endoderme do tipo fi. No entanto, Wilcox (1962) demonstrou que em Libocedrus decurrens Torr. o espessamento em fi não era suberizado, o que o diferenciava funcionalmente das estrias de Caspary quanto a permeabilidade de parede. Essa sugestão foi confirmada pelos estudos de Peterson et al. (1981), que testaram o movimento apoplástico de Trinopal CBS - X, um corante de baixo peso molecular, 562,6, em raízes de Pyrus malus L. e Pelargonium hortorum L.H. Bailey, e constataram uma impermeabilidade da endoderme e uma permeabilidade do espessamento em fi, em ambas as espécies, para moléculas de peso molecular relativamente baixo, a exemplo do corante testado. A permeabilidade do espessamento em fi também foi comprovada para o corante calcoflúor em estudos realizados por Perumalla et al. (1990) com diferentes espécies de angiospermas. Experimento similar foi realizado com raízes de Zea mays L., utilizando o corante hemisulfato de berbenina, ao qual a exoderme foi impermeável e o espessamento em fi da rizoderme permeável a sua penetração (Degenhardt \& Gimler 2000). No entanto, Fernandez-Garcia et al. (2009), estudando a permeabilidade de espessamentos em fi em raízes de Brassica oleracea L. cv. Maratón, cultivada em ambiente com alta salinidade, utilizando $\mathrm{LaCl}_{3}$ observaram a interferência do espessamento em fi dificultando o movimento apoplástico de $\mathrm{La}^{3+}$, assim como também foi observada maior facilidade para a passagem de $\mathrm{K}^{+}$em detrimento de $\mathrm{Na}^{+}$em camadas celulares fi-espessadas, evidenciando, portanto, uma efetiva seletividade para o movimento de íons.

\section{Variadas funções do espessamento em fi}

O espessamento em fi, embora venha sendo estudado há mais de cem anos, ainda é considerado uma estrutura enigmática do ponto de vista funcional, o que tem instigado quase todas as pesquisas realizadas sobre o assunto em dar respostas quanto à sua função através de estudos de localização e desenvolvimento, estrutura e composição química, sistemática ou ecologia.

Devido a precoce lignificação do espessamento em fi em porções frontais ao floema dos tecidos radiculares em diferenciação, quando ainda da ausência de suberização e lignificação da endoderme, tem sido atribuído a ele um papel de obstáculo mecânico que retardaria, apesar da sua permeabilidade, um refluxo de água da região do cilindro central ao córtex (Pratikakis et al. 1998) ou mesmo poderia atuar como uma força mecânica que suportaria as pressões interpostas exercidas pelas células que ainda estão em processo de expansão no cilindro central e no córtex. Passioura (1988) também atribui as camadas fi-espessadas uma resistência mecânica a retração diurna da raiz crescendo em um solo seco, efeito este que poderia reduzir a habilidade de absorção de água.

Espessamentos lignificados, proeminentes e irregulares em parede, a exemplo do espessamento em fi, têm sido relacionados a presença de metais pesados no meio de cultivo ou a capacidade de acúmulo desses metais pelas plantas. Foi observada uma ocorrência natural desses espessamentos de parede em raízes de Thlaspi caerulescens J. Presl $\&$ C. Presl, uma espécie hiper-acumuladora de metais, 
em detrimento da sua ausência na espécie de mesmo gênero, mas não acumuladora, Thlaspi arvense L. (Broadley et al. 2007). Degenhardt \& Gimler (2000) estudando raízes de Zea mays L. observaram que ocorreu formação de camadas descontínuas de células fi-espessadas no terço superior da rizoderme das raízes mais velhas e somente em plantas cultivadas utilizandose escória como substrato, a qual dificultava a penetração de raízes, apresentava característica alcalina e continha sais e metais pesados em alta concentração. A descontinuidade dos espessamentos na rizoderme sugere que o surgimento dos espessamentos, para este caso particular, não foi relevante na formação de uma barreira apoplástica a penetração de solutos, mas que poderia atuar como suporte mecânico contra rompimento de tecidos em decorrência do alto grau de impedimento mecânico do substrato.

A evidência de suporte mecânico do espessamento em fi a penetração de raízes é também suportada pelo estudo de Christodoulakis \& Psaras (1987), no qual foi observado que Ceratonia siliqua L., quando cultivada em perlita saturada com solução de Hoagland, não desenvolvia espessamentos em fi, diferentemente do que ocorria quando cultivada em solo. Soukup et al. (2004) também observaram que diferentemente do que ocorria na condição de solo, Prunus avium L. não desenvolvia espessamento em fi quando cultivada in vitro. Evidências relacionadas ao habitat natural que indicam que o espessamento em fi pode ser importante no processo de penetração de raízes foram relatadas por Larson et al. (2000), os quais observaram que 20 dentre 26 espécies de gimnospermas estudadas, de ocorrência natural em solo com elevado grau de impedimento à penetração de raízes, apresentavam espessamento em fi.

Outra função, também relacionada a suporte mecânico, e atribuída ao espessamento em fi é quanto a proteção contra a invasão de agentes biológicos as camadas mais profundas do córtex ou ao cilindro central. Em Dryas integrifolia Vahl o surgimento de espessamento em fi ocorreu em resposta à interação micorrízica para com o fungo Hebeloma cylindrosporum Romagnesi. Os pesquisadores observaram que o desenvolvimento da rede de Hartig em direção às camadas mais profundas do córtex era interrompido pelas camadas fi-espessadas e nas regiões onde a rede de Harting não era formada não havia o desenvolvimento dos espessamentos em fi (Melville et al. 1987). Resultados similares quanto a função de barreira estrutural do espessamento em fi foram observados por Wilcox \& Wang (1987), os quais constataram que a segunda camada cortical fi-espessada de Betula alleghaniensis Britton bloqueou o desenvolvimento cortical da rede de Hartig oriunda da interação micorrízica com o fungo Chloridium paucisporum Wang. Em alguns outros estudos de interação micorrízica têm-se percebido a presença dos espessamentos em fi nas células corticais, no entanto não foram realizados estudos de correlação destes como barreira mecânica a penetração do fungo (Massicotte et al. 1999, Massicotte et al. 1988).

Além dos estudos que indicam os espessamentos em fi como barreira mecânica a penetração de fungos foi observado em Ceratonia siliqua L. (Pratikakis et al. 1998) e em Quercus coccifera L. (Christodoulakis \& Psaras 1988) que estes espessamentos podem conter taninos e, portanto, atuar também como uma barreira química a invasão de microrganismos.

As descobertas mais recentes que se referem a função do espessamento em fi têm respaldado a sugestão inicial de Russow (1875) e Van Tieghem (1888), os quais atribuíram a essa estrutura uma função de suporte mecânico. No entanto seu desenvolvimento em espécies acumuladoras de metais pesados ou que estejam sob estresse salino (Broadley et al. 2007, Degenhardt \& Gimler 2000, FernandezGarcia et al. 2009) mantém a hipótese de sua atuação na seleção de íons. Tajima et al. (2008), trabalhando com uma espécie que apresenta nódulos nas raízes, em função da associação com bactérias que atuam na fixação biológica de nitrogênio, Arachis hypogaea L., embora não tenham sugerido uma função específica para os espessamentos em fi e outros espessamentos encontrados nesta espécie, supõem, baseandose no posicionamento destes no órgão, que haja outras funções para os espessamentos além de simples suporte mecânico para a relação planta-bactéria.

\section{Espessamento em fi e taxonomia}

$\mathrm{O}$ espessamento em fi tem sido utilizado como caractere anatômico-radicular para estudos de sistemática. Os primeiros estudos haviam identificado a sua presença em várias espécies como Cycas circinalis L., Torreya nucifera Siebold \& Zucc., Juniperus sp. Thuja sp., Araucaria sp., Sequoiadendron giganteum J. Buchholz, Ginkgo 
biloba L., Taxus baccata L., Herpestis monniera Kunth (Van Tieghem 1871, Van Tieghem 1888, Duval 1906 apud Solereder 1908). Bergendal (1883) e Van Tieghem \& Monal (1888) observaram a ocorrência de espessamento em fi em três gêneros de Geraniaceae e Lindinger (1908) observou sua presença em várias famílias de monocotiledôneas. Noelle (1910) passou a atribuir um caráter taxonômico aos estudos de espessamento em fi, especialmente em gimnospermas, diagnosticando-o e usando-o para identificação das famílias Araucariaceae, Cupressaceae e Taxodiaceae. Esse foco taxonômico tem se estendido até a época atual podendo ser observado nas pesquisas de Pires et al. (2003), Gerrath et al. (2002) e Brundrett et al. (1990).

Alguns pesquisadores têm realizado experimentos no intuito de confirmar a característica constitutiva do espessamento em fi. Para algumas espécies o espessamento em fi continua sendo considerado um caractere constitutivo, a exemplo de sua presença em Cryptomeria japonica D. Don., ou de sua ausência em Pinus rigida Mill. e Pinus aristata Engel, independentemente das condições de cultivo, se normais, de alagamento ou de compactação do solo, embora tenha ocorrido diminuição da área ocupada pelos espessamentos nas condições de alagamento e compactação (Gerrath et al. 2005), enquanto que para outras espécies como Brassica oleracea L. e Eriobotrya japonica Lindl tem sido observado aumento em número e área ocupado pelos espessamentos em fi em condições de deficiência hídrica ou de salinidade (Pan et al. 2006, Lopez-Perez et al. 2007).

Várias pesquisas revelam a fragilidade da generalização do conceito de constitutivo ou não constitutivo do espessamento em fi para as diferentes espécies. Millay et al. (1987) relatam que já havia a presença do espessamento em fi em fósseis vegetais triássicos provenientes da Antártica. No entanto, pela presença atual dessa mesma característica em gimnospermas e angiospermas estes pesquisadores atribuem a essa estrutura um caráter muito mais ecológico do que de significação filogenética. Nos estudos desenvolvidos por Gerrath et al. (2002) foi observado que uma única espécie, dentre nove estudadas da família Pinaceae apresentava espessamento em fi e que, portanto, a presença ou ausência desse caractere não poderiam ser utilizadas como característica de família. Além disso, a possibilidade de indução da formação do espessamento em fi, como já relatado previamente, como resposta a condições do ambiente (Christodoulakis \& Psaras 1987, Degenhardt \& Gimler 2000, Melville et al. 1987), não permite considerá-lo como um caractere estritamente constitutivo, característica fundamental para sua aplicação em classificação taxonômica.

\section{Conclusões}

O espessamento em fi, apesar de ser conhecido há mais de um século, tem sido alvo de controvérsias ao longo do tempo quanto ao seu papel funcional.

$\mathrm{O}$ conjunto dos resultados das pesquisas sobre o tema revela que o espessamento em fi pode atuar tanto como uma estrutura de suporte mecânico, contribuindo para integridade e funcionalidade das raízes, mesmo antes da diferenciação de outros tecidos que podem participar da sustentação mecânica, quanto ainda ter um papel fisiológico de seletividade em relação ao movimento apoplástico de íons na raiz.

Ultimamente a importância do espessamento em fi tem sido desconsiderada como caractere filogenético, mas tem sido aumentada quanto à significação ecológica.

Vislumbra-se que as futuras pesquisas sobre espessamento em fi não procurem excluir sua função fisiológica em detrimento da estrutural ou vice-versa, mas que possam aceitar a hipótese da co-existência de múltiplas funções.

Espera-se que novos trabalhos de pesquisa contribuam para a ampliação e/ou solidificação do entendimento do papel funcional do espessamento em fi quanto a proteção da planta a infecção por agentes com ou sem potencialidade patogênica ou ao acúmulo de minerais em tecidos radiculares que representem ou não risco de toxicidade, assim como ajudem a responder a intrigante questão da penetração de raízes em algumas espécies em substratos com alto grau de impedimento mecânico.

\section{Literatura citada}

Bergendal, D. 1883. Undersokningar ofver Geraniaceernas byggnad med nagra jemforande blickar pa andra Gruinaler. Lunds Universitets Ars-skrift 19: 23-134.

Bona, C. 1999. Adaptações morfo-anatômicas dos órgãos vegetativos de Bacopa salzmanii (Benth.) Wettst. ex Edwall e Bacopa monnierioides (Cham.) Robinson (Scrophulariaceae) em ambiente terrestre e aquático. Tese de Doutorado, Universidade de São Paulo, São Paulo. 
Bona, C. \& Morretes, B.L. 2003. Anatomia das raízes de Bacopa salzmanii (Benth.) Wettst. ex Edwall e Bacopa monnierioides (Cham.) Robinson (Scrophulariaceae) em ambientes aquático e terrestre. Acta Botanica Brasilica 17: $155-170$.

Boreau, E. 1939. Recherches antaomiques et expérimentales sur lóntogénie des plantules des Pinacées et ses rapports avec la phylogéni. Annales des Sciences Naturelles Botanique 1: 1-219.

Broadley, M.R., White, J.P., Hammond, J.P., Zelko, I. \& Lux, A. 2007. Zinc in plants. New Phytologist 173: 677-702.

Brundrett, M., Murase, G. \& Kendrick, B. 1990. Comparative anatomy of root and mycorrhizae of common Ontario trees. Canadian Journal of Botany 68: $551-578$

Christodoulakis, N.S. \& Psaras, G.K. 1987. Stomata on the primary root of Ceratonia siliqua. Annals of Botany 60:295-297.

Christodoulakis, N.S. \& Psaras, G.K. 1988. A contribution to root study of the evergreen sclerophylls. Anatomy of the primary root of Quercus coccifera L. Flora 180: 445-453.

Degenhardt, B. \& Gimmler, H. 2000. Cell wall adaptation to multiple environmental stress in maize roots. Journal of Experimental Botany 51: 595-603.

Esau, K. 1943. Vascular differentiation in the pear root. Hilgardia 15: 299-324.

Fernandez-Garcia, N., Lopez-Perez, L., Hernandez, M. \& Olmos, E. 2009. Role of phi cells and the endodermis under salt stress in Brassica oleracea. New Phytologist 181:347-360.

Gerrath, J.M., Covington, L., Doubt, J. \& Larson, D.W. 2002. Occurrence of phi thickenings is correlated with gymnosperm systematics. Canadian Journal of Botany 80: $852-860$

Gerrath, J.M., Mattheus, U., Purich, M. \& Larson, D.W. 2005. Root environmental effects on phi thickening production and root morphology in three gymnosperm. Canadian Journal of Botany 83: 379-385.

Haas, D.L., Carothers, Z.B. \& Robbins, R.R. 1976. Observations on the phi thickenings and caparian strips in Perlagonium roots. American Journal of Botany 63: 863-867.

Larson, D.W., Matheus, U. \& Kelly, P. 2000. Cliff ecology. Pattern and process in cliff ecosystems. Cambridge University Press, Cambridge.

Lindinger, L. 1906. Zur Anatomie und Biologie der Monokotylenwurzel - Beihefte Zum Botanischen Centralblatt: 19: 1.

Lopez-Perez, L., Fernandez-Garcia, N., Olmos, E. \& Carvajal, M. 2007. The phi thickening in roots of broccoli plants: an acclimatation mechanism to salinity. International Journal of Plant Science 168: 1141-1149.
Mackenzie, K.A.D. 1979. The development of the endodermis and phi layer of aplle roots. Protoplasma 100: 21-32.

Massicotte, H.B., Peterson, R.L., Ackerley, C.A. \& Melville, L.H. 1988. Structure and ontogeny of Betula alleghaniensis - Pisolithus tinctorius ectomycorrhizae. Canadian Journal of Botany 68: 579-593.

Massicotte, H.B., Melville, L.H., Peterson, R.L. \& Unestam, T. 1999. Comparative studies of ectomycorrhiza formation in Alnus glutinosa and Pinus resinosa with Paxillus involutus. Michorrhiza 8: 229-240.

Melville, L.H., Massicote, H.B. \& Peterson, R.L. 1987. Ontogeny of early stages of ectomycorrhizae synthesized between Dryas integrifolia and Hebeloma cylindrosporum. Botanical Gazette 148: 332-341.

Millay, M.A., Taylor, T.N. \& Taylor, E.L. 1987. Phi thickenings in fossil seed from Antarctica. IAWA Bulletin 8: 191-201.

Noelle, W. 1910. Studien zur vergleichenden anatomie und morphologie der koniferenwurzeln mit rucksicht auf die systematic. Botanic Zeitung 68: 169-266.

Pan, C.X., Nakao, Y. \& Nii, N. 2006. Anatomical development of phi thickening and the casparian strip in loquat roots. Journal of the Japanese Society of Horticultural Science 75: 445-449.

Perumalla, C.J., Peterson, C.A. \& Enstone, D.E. 1990. A survey of angiosperm species to detect hypodermal Casparian bands. I. Roots with a unisseriate hypodermis and epidermis. Botanical Journal of Linnean Society 103: 93-112.

Passioura, J.B. 1988. Waster transport in and to roots. Annual Review of Plant Physiology and Plant Molecular Biology 39: 245-265.

Peterson, C.A. \& Cholewa, E. 1998. Structural modifications of the apoplast and their potential impact on ion uptake. Zeitschrift für Pflanzenernährung und Bodenkunde 161: 521-531.

Peterson, C.A., Emanuel, M.E. \& Weerdenburg, C.A. 1981. The permeability of phi thickenings in apple (Pyrus malus) and geranium (Perlagonium hortorum) roots to an apoplastic fluorescent dye tracer. Canadian Journal of Botany 59: 1107-1110.

Peterson, C.A. \& Perumalla, C.J. 1990. A survey of angiosperm species to detect hypodermal casparian bands. II. Roots with a multiseriate hypodermis or epidermis. Botanical Journal of the Linnean Society 103: 113-125.

Pires, M.F.O., Semir, J., Pinna, G.F.A.M. \& Felix, L.P. 2003. Taxonomic separation of the genera Prosthechea and Encyclia (Laeliinae: Orchidaceae) using leaf and root anatomical features. Botanical Journal of the Linnean Society 143: 293-303.

Pratikakis, E., Rhizopoulou, S. \& Psaras, G.K. 1998. A phi layer in roots of Ceratonia siliqua L. Botanica Acta 111:93-98. 
Riedhart, J.M. \& Guard,A.T. 1957. On the anatomy of the roots of apple seedlings. Botanical Gazette 118: 191-194.

Russow, E. 1875. Betrachtungen uber das Leitbundel und Grundgewebe aus vergleichend morphologishem und phylogentischem Gesischstpunkt. Schnakenburg's Anstalt. Dorpat.

Solereder, H. 1908. Systematic anatomy of the Dicotyledons. v. 2. Claredon Press, Oxford.

Soukup, A., Malá, J., Hrubcová, M., Kálal, J. \& Votrubová, O. 2004. Differences in anatomical structure and lignin content of roots of pedunculate oak and wild cherrytree plantlets during acclimatation. Biologia Plantarum 48: 481-489.

Tajima, R., Abe, J., Lee, O., Morita, S. \& Lux, A. 2008. Developmental changes in peanut root structure during root growth and root-structure modification by nodulation. Annals of Botany 101: 491-499.

Van Fleet, D.S. 1961. Histochemistry and function of the endodermis. The Botanical Review 27: 165-220.

Van Tieghem, P. 1871. Mémoire sur la racine. Annales des Science Naturelles (Paris) 13: 187-195.
Van Tiehgem, P. 1888. Le réseau de soutien de l'ecorce de la racine. Annales des Sciences Naturelles, Botanique Series 7/8: 375-378.

Van Tieghem, P. \& Monal. 1888. Sur le réseau sousépidermique de la racine des Géraniacées. Bulletin de la Société Botanique de France 35: 274.

Weerdenburg, C.A. \& Peterson, C.A. 1983. Structural changes in phi thickenings during primary and secondary growth in roots. 1. Apple (Pyrus malus) Rosaceae. Canadian Journal of Botany 61: 2570-2577.

Wilcox, H. 1962. Growth studies of the root of incense cedar, Libocedrus decurrens. I. The origin and development of primary tissues. American Journal of Botany 49: 221-236.

Wilcox, H. \& Wang. C.J.K. 1987. Michorrizal and pathological associations of dematiaceous fungi in roots of seven-month-old tree seedlings. Canadian Journal of Forest Research 17: 884-899.

Wilson, C.A. \& Peterson, C.A. 1983. Chemical composition of the epidermal, hypodermal, endodermal and intervening cortical cells walls of various plant roots. Annals of Botany 52: 759-769. 